\section{Vehicle optimal road departure prevention via model predictive control}

Hongliang Yuan ${ }^{1}$, Yangyan Gao ${ }^{2}$ and Timothy J Gordon ${ }^{2}$
Journal Title

$\mathrm{XX}(\mathrm{X}): 1-10$

(C) The Author(s) 2015

Reprints and permission:

sagepub.co.uk/journalsPermissions.nav

DOI: 10.1177/ToBeAssigned

www.sagepub.com/

@SAGE

\begin{abstract}
This article addresses the problem of road departure prevention using integrated brake control. The scenario considered is when a high speed vehicle leaves the highway on a curve and enters the shoulder or another lane, due to excessive speed, or where the friction of the road drops due to adverse weather conditions. In such a scenario, the vehicle speed is too high for the available tyre-road friction and road departure is inevitable; however, its effect can be minimized with an optimal braking strategy. To achieve online implementation, the task is formulated as a receding horizon optimization problem and solved in a linear model predictive control (MPC) framework. In this formulation, a nonlinear tire model is adopted in order to work properly at the friction limits. The optimization results are close to those obtained previously using a particle model optimization, PPR, coupled to a control algorithm, MHA, specifically designed to operate at the vehicle friction limits. This shows the MPC formulation may equally be effective for vehicle control at the friction limits. The major difference here, compared to the earlier PPR/MHA control formulation, is that the proposed MPC strategy directly generates an optimal brake sequence, while PPR provides an optimal reference first, then MHA responds to the reference to give closed-loop actuator control. The presented MPC approach has the potential to be used in future vehicle systems as part of the overall Active Safety Control (ASC) to improve overall vehicle agility and safety.
\end{abstract}

\title{
Keywords
}

Vehicle dynamics, model predictive control, vehicle safety system, vehicle braking control, passenger vehicles

\section{Introduction}

In recent decades, the development of Advanced Driver Assistance Systems (ADAS) has received much attention. This is broadly considered as an effective approach to meet many challenges such as passenger safety and comfort, fuel economy, and emission reduction, etc. Examples include the well known anti-lock brake systems (ABS) and the electronic stability program (ESP) to help vehicle stabilisation via global chassis control; another example is adaptive cruise control (ACC) which can help a vehicle adjust speed in response to traffic conditions. A very recent development is in the area of collision avoidance and crash mitigation systems; examples include the Mercedes-Benz Pre-safe brake and Volkswagen's Front Assist and City Emergency Brake system, ${ }^{1}$ which have shown the effectiveness of helping the driver avoid rear-end collisions at low speeds by automatically braking when a potential collision is detected.

Over-speed in curves may result in road departure and interference with oncoming traffic. Two US reports on fatal single-vehicle run-off road crashes ${ }^{2 ; 3}$ show that around onethird of such crashes occurred in a turn and nearly half of these crashes involved speeding. Furthermore, the reports conclude that run-off road crashes are more likely to occur in adverse weather conditions. Therefore an active control strategy for braking is needed in the ADAS functionality to minimise the likelihood and effects of imminent road departure.

To minimize the risk or extent of road departure, intuitively, the vehicle needs to maximize its path curvature while maintaining its yaw stability. There is a clear link to the problem of terminal understeer where, at the limits of road friction, vehicle path curvature reaches an upper

\footnotetext{
1 Tongji University, China

2 University of Lincoln, UK
}

\section{Corresponding author:}

Timothy J Gordon, School of Engineering, University of Lincoln, Lincoln, LN6 7TS, UK.

Email: TGordon@lincoln.ac.uk 
limit. It is well known that for oversteer compensation, the yaw instability can be effectively suppressed by applying braking forces to generate yaw moments acting in the opposite direction of turn, hence the excessive yaw rate and the body sideslip are simultaneously reduced. Earlier work on understeer compensation has adopted the approach of imposing a yaw control (YC) opposite to the oversteer compensation by differential braking. ${ }^{4-6}$ However, this approach does not consider the combined slip effect of the tyres, where braking force would reduce the available lateral force at the friction limit. Hence the yaw control itself can not be guaranteed to increase the path curvature; moreover, it is possible to induce oversteer due to increasing the yaw angle and simultaneously inducing large body side-slip angles.

More significantly, to increase the curvature of the vehicle trajectory, researchers have realized that it is highly effective to include a control component to reduce vehicle speed. ${ }^{7}$ In essence, this requires an integrated strategy controlling the longitudinal and lateral forces as a whole. Therefore optimization of braking and lateral forces is necessary to minimize the maximum deviation from the intended path. For such an objective, the parabolic path reference (PPR) is obtained offline based on Pontryagin's minimum principle under certain assumptions, which allows speed reduction and progressively increasing path curvature. ${ }^{8 ; 9}$ The optimization problem then reduces to one of tracking the PPR reference using suitable torque distribution techniques, and to this end a new optimal torque distribution strategy, called the Modified Hamiltonian Algorithm (MHA) (also based the minimum principle), has recently been proposed. ${ }^{10-12}$

Elsewhere, model predictive control (MPC) ${ }^{13-19}$ has become more frequently applied in vehicle dynamics control. Incorporating a receding horizon technique, MPC can provide online solutions with certain (limited) guarantees of optimality for multi-input-multi-output (MIMO) systems. Furthermore, MPC provides a systematic way of handling state and input constraints, which is desirable for many realworld applications. Though MPC have been proven to be effective in many non-critical applications, it is interesting to consider how MPC performs in vehicle control at the limits of friction. In the application of road departure prevention, the problem is close to earlier investigations mentioned above $^{8-10}$; here we propose using MPC to address the same scenario. This is based on the understanding that MPC, once tested in the situation of quasi-static braking and cornering, it may be easily extended to other, more complex scenarios. The MPC formulation will adopt a similar objective function as presented in earlier literature, then to convert the problem to be an online finite horizon optimization. Nonlinear tire

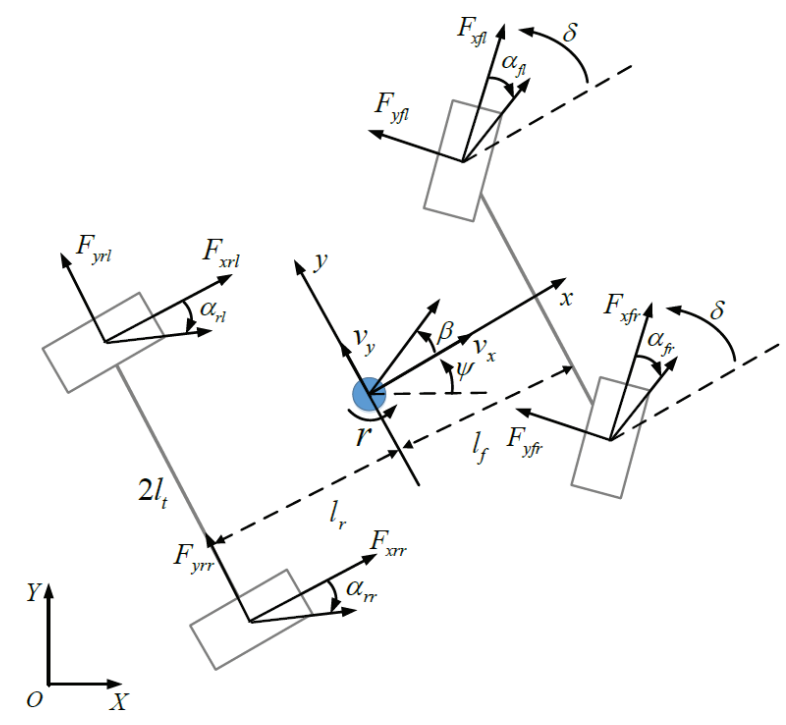

Figure 1. The two-track vehicle model. The arrows show the positive direction of each angle or force. Non-labelled arrows indicate the local velocity vector.

forces modeled with the well-known Pacejka magic formula are considered. From this, the MPC controller actively distributes the four wheel braking torque.

The remainder of the paper is organized as follows: the second section defines the two-track nonlinear vehicle model, and the third section formulates an MPC-based vehicle chassis control system using differential braking. Simulations and comparative studies will be presented in the fourth section. Conclusions are given in the fifth section.

\section{The vehicle model}

The vehicle model used for control development is a 3-degree-of-freedom (DOF) planar two-track model with 6 states $v_{x}, v_{y}, r, \psi, X, Y$, where $v_{x}, v_{y}, r$ are the longitudinal velocity, lateral velocity and yaw rate in the vehicle body axis system, and $\psi, X, Y$ are respectively the yaw angle, and mass-centre cartesian coordinates in the global coordinate system. The vehicle's front steering (road wheel) angle will be presumed set by a simple driver input, namely a step-steer with magnitude determined by the low speed geometry - see Table 2 below. By contrast, the brake controller is assumed to have full authority of the braking torque and to have access to appropriate sensors.

The planar vehicle model is shown in Figure 1, where $F_{x f l}, F_{x f r}, F_{x r l}, F_{x r r}$ are the longitudinal forces of front-left, front-right, rear-left, rear-right tires respectively. Similarly, $F_{y f l}, F_{y f r}, F_{y r l}, F_{y r r}$ are the lateral forces of 4 tires and $\alpha_{f l}, \alpha_{f r}, \alpha_{r l}, \alpha_{r r}$ are tire lateral slip angles. $l_{f}$ and $l_{r}$ are the distance from the vehicle $C G$ to the front and rear axle respectively; $l_{t}$ is half of the track width. The variables $v_{x}$ and $v_{y}$ are respectively the longitudinal and 
lateral velocity in the vehicle body frame. Further, $\delta$ is the front road wheel angle, and $\beta$ is the body slip angle, $r$ is the vehicle yaw rate, and $\psi$ is the yaw angle measured from the $X$ axis of the global coordinate system. Using these notations, the motion dynamics are described by the following equations:

$m\left(\dot{v}_{x}-v_{y} r\right)=F_{x f l} \cos \delta-F_{y f l} \sin \delta+F_{x f r} \cos \delta-F_{y f r}$

$\cdot \sin \delta+F_{x r l}+F_{x r r}$

$m\left(\dot{v}_{y}+v_{x} r\right)=F_{x f l} \sin \delta+F_{y f l} \cos \delta+F_{x f r} \sin \delta+F_{y f r}$

$\cdot \cos \delta+F_{y r l}+F_{y r r}$

$I_{z z} \dot{r}=\left(F_{x f r} \cos \delta-F_{y f r} \sin \delta+F_{x r r}-F_{x f l} \cos \delta+F_{y f l}\right.$

$\left.\cdot \sin \delta-F_{x r l}\right) \cdot l_{t}+\left(F_{x f l} \sin \delta+F_{y f l} \cos \delta+F_{x f r} \sin \delta\right.$

$\left.+F_{y f r} \cos \delta\right) \cdot l_{f}-\left(F_{y r l}+F_{y r r}\right) \cdot l_{r}$

$\dot{\psi}=r$

$\dot{X}=v_{x} \cos \psi-v_{y} \sin \psi$

$\dot{Y}=v_{x} \sin \psi+v_{y} \cos \psi$

\section{The tire model}

In the above model equations (1), the front road wheel angle $\delta$ will be a suitable step-steer, determined from the low-speed Ackerman condition - see Table 2, and therefore held constant during the event. The longitudinal tire forces and lateral tire forces can not be separately used as control inputs since they are not independent. It is preferred to use longitudinal tire forces as inputs since they can be regulated by the brake torque, therefore it is desirable to eliminate the lateral forces in the model (1). To achieve this, the friction ellipse relationship between longitudinal and lateral tire forces are assumed, which is illustrated in Figure 2.

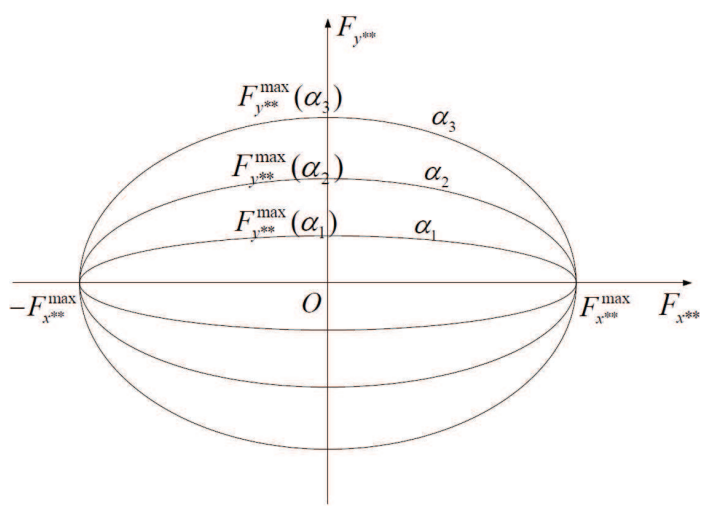

Figure 2. The elliptical relationship between longitudinal and lateral tire forces at different slip angles.
For each tire, the equation of the friction ellipse is given by:

$$
\left(\frac{F_{x * *}}{F_{x * *}}\right)^{2}+\left(\frac{F_{y * *}}{F_{y * *}}\right)^{2}=1
$$

where the subscript $* *$ denotes $f l, f r, r l, r r$. In the friction ellipse equation (2), the maximum achievable longitudinal force is always $F_{x * *}^{\max }=\mu F_{z * *}$, where $\mu$ is the road friction coefficient and $F_{z * *}$ is the vertical load of the corresponding tire. The maximum lateral $F_{y * *}^{\max }$ (achieved when no longitudinal slip) varies with tire slip angle, and can be given by the Pacejka magic formula ${ }^{20}$ :

$$
F_{y * *}^{\max }(\alpha)=\mu F_{z * *} \cdot M(\alpha)
$$

with

$$
M(\alpha)=D \sin \left\{C \tan ^{-1}\left[B \alpha-E\left(B \alpha-\tan ^{-1} B \alpha\right)\right]\right\}
$$

where $B, C, D, E$ are parameters of the magic formula. Substituting equation (3) into equation (2), the lateral forces of combined slip can be expressed by the longitudinal forces:

$$
F_{y * *}=M(\alpha) \cdot \sqrt{\left(\mu F_{z * *}\right)^{2}-F_{x * *}^{2}}
$$

Thus equation (4) is used to eliminate the lateral tire forces in model (1). In this study, the magic formula parameters $B, C, D, E$ for the tire model (4) were fitted via the least square error (LSE) criteria using CarSim $^{21}$ tire data for the tire $215 / 55 R 17$. The fitting parameters are shown in Table 1.

According to Table 1, the parameter $E$ is quite small for all vertical loads, so for simplicity we use the approximation $E=0$. Further analysis shows that parameters $B, C, D$ have certain linearity with respect the vertical loads as shown in Figure 3. Therefore, they are further fitted as linear equations:

$$
\begin{aligned}
& B=-1.4758 \times 10^{-4} F_{z}+13.0409 \\
& C=7.4666 \times 10^{-7} F_{z}+1.4465 \\
& D=-9.0695 \times 10^{-6} F_{z}+1.0161
\end{aligned}
$$

Figure 3 includes a comparison of pure lateral force provided by CarSim tire data together with the calculated force from equation (4); it shows that they match quite well. It is also necessary to test equation (4) for other conditions; here we choose a combined slip situation where the longitudinal tire force is nonzero. Since CarSim only directly provides force data for pure side slip, to do this verification, we set a double lane change maneuver with $120 \mathrm{~km} / \mathrm{h}$ on a surface with friction coefficient 0.4 , and the 
Table 1. $B, C, D, E$ at different vertical loads

\begin{tabular}{lllllllll}
\hline \hline$F_{z}(N)$ & 1594 & 3187 & 4781 & 6374 & 7968 & 9561 & 11155 & 12749 \\
$B$ & 12.7603 & 12.5691 & 12.3569 & 12.1300 & 11.8914 & 11.6432 & 11.3866 & 11.1227 \\
$C$ & 1.4481 & 1.4490 & 1.4499 & 1.4510 & 1.4522 & 1.4535 & 1.4549 & 1.4564 \\
$D$ & 0.9988 & 0.9871 & 0.9741 & 0.9601 & 0.9455 & 0.9302 & 0.9144 & 0.8982 \\
$E$ & $0.0293 \mathrm{e}-15$ & $0.0603 \mathrm{e}-15$ & $0.0387 \mathrm{e}-15$ & $0.0033 \mathrm{e}-15$ & $0.1406 \mathrm{e}-15$ & $0.0347 \mathrm{e}-15$ & $0.0107 \mathrm{e}-15$ & $0.0004 \mathrm{e}-15$ \\
$L S E(N)$ & 5.0962 & 10.0173 & 14.7306 & 19.2219 & 23.4742 & 27.4771 & 31.2175 & 34.6881 \\
\hline
\end{tabular}
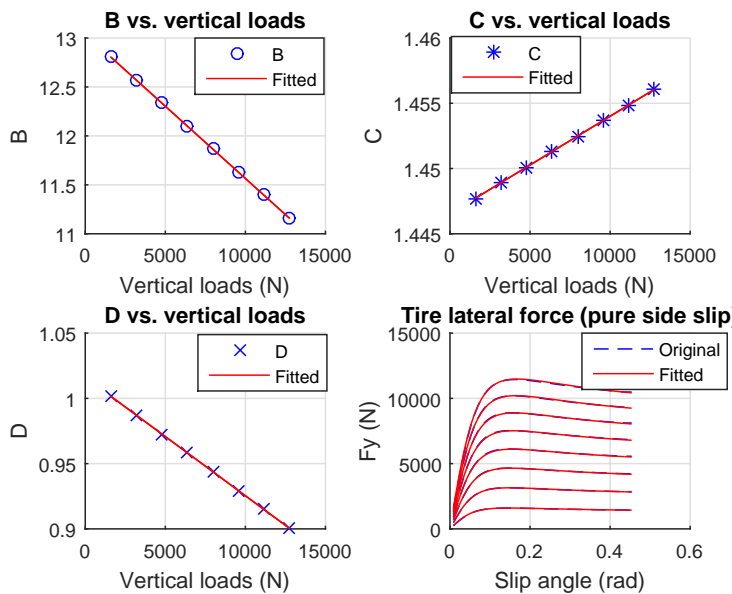

Figure 3. LSE fitted results

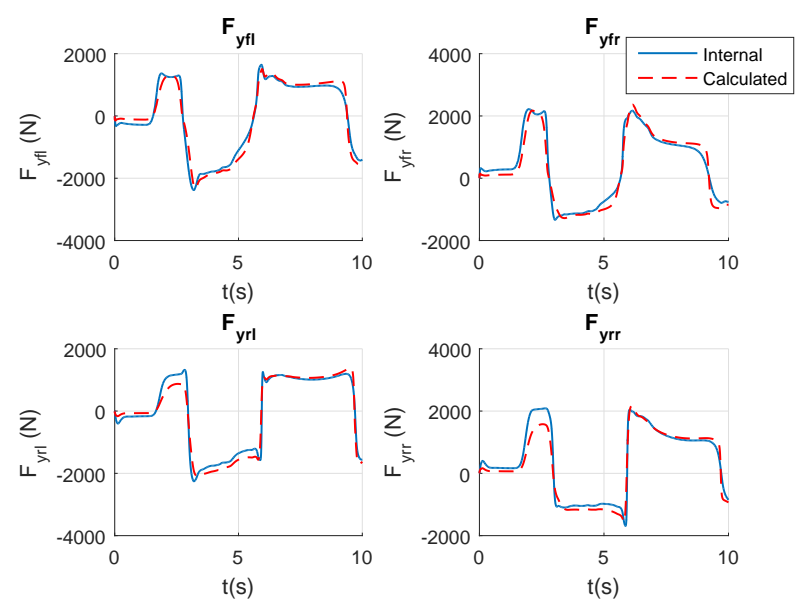

Figure 4. Internal lateral tire force and calculated tire force with equation (4) under a DLC maneuver

internal lateral force of the software and the calculated lateral force for four wheels are compared in Figure 4. It shows that equation (4) reproduces the lateral forces quite well in this more complex handling situation. Note that this validation step is only for controller design and is not expected to be fully accurate; performance evaluation will however make use of the full CarSim tyre model.

\section{Road departure prevention}

We consider the following scenario: a high speed vehicle is starting to depart the highway on a curved segment due to excessive entry speed by the driver or because the friction of the road is less than expected. Let $L=l_{f}+l_{r}$ be the wheelbase; then the reference path radius can be expressed as: $R_{r e f}=L / \delta$. The maximum achievable longitudinal speed $v_{\text {lim }}$ is jointly determined by the path radius and the road friction coefficient $\mu$ with the following equation:

$$
v_{l i m}=\sqrt{\mu g R_{r e f}}
$$

where $g$ is the gravity constant. When the vehicle's initial speed $v_{0}$ is greater than $v_{\text {lim }}$ at the beginning of the event, deviation from the circular reference path becomes inevitable. At the starting point, one might intuitively expect to brake the vehicle hard; however this action reduces the lateral force the vehicle can achieve so that it can actually increase the overall off-tracking. On the other hand, if one does not brake the vehicle at all, the vehicle can concentrate all friction force in the lateral direction; in this case however, the off-tracking will also be high due maintaining high speed throughout the manoeuvre. Thus, it is intuitively clear that the road departure mitigation problem is required to determine the optimal tradeoff between speed reduction and cornering acceleration. This scenario was previously considered and solved using a combination of offline particle optimization (PPR) and online parameter tuning; ${ }^{9}$ as mentioned, as well as the more general MHA method; in this paper we take an independent approach to minimizing the off-tracking, i.e. by developing a novel implementation of MPC control.

The scenario is illustrated in Figure 5, where $O X Y$ is the global coordinate system, the dotted curve is the desired circular path with radius $R_{r e f}$, and $M$ is the center of circular path with coordinates $\left(0, Y_{M}\right)$. The solid curve represents the actual path of the vehicle $C G$; at an unspecified time $T$, the distance between the center of curved path and the vehicle $C G$ reaches its maximum $H_{\max }$, where

$$
H_{\max }=\sqrt{X_{T}^{2}+\left(Y_{T}-Y_{M}\right)^{2}} .
$$

According to the Ackerman steering condition mentioned above, the step-steer magnitude is set to:

$$
\delta_{H}=\frac{i_{S} L}{R_{r e f}},
$$




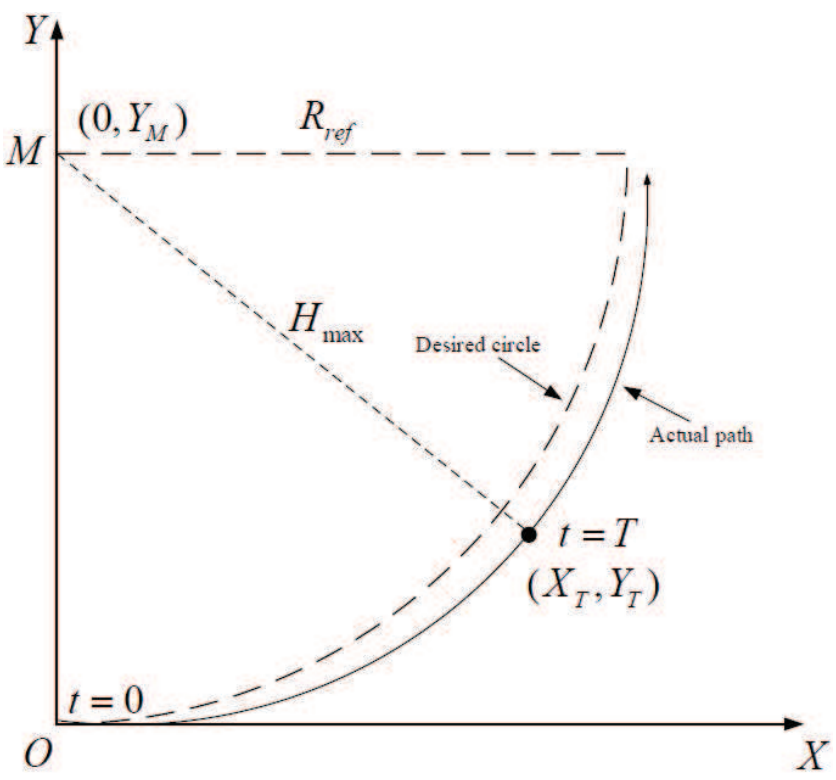

Figure 5. Scenario of road departure mitigation

where $\delta_{H}$ is the handwheel angle and $i_{S}$ is the steering ratio. Denote

$$
\begin{aligned}
& \xi=\left[v_{x} v_{y} r \psi X Y\right]^{T} \\
& u=\left[\begin{array}{llll}
F_{x f l} & F_{x f r} & F_{x r l} & F_{x r r}
\end{array}\right]^{T}
\end{aligned}
$$

Then the road departure mitigation problem can be described as:

$$
\begin{array}{ll} 
& \min _{u \leq 0} H_{\max } \\
\text { s.t. } & \dot{\xi}=f(\xi, u) \\
& \xi(0)=\xi_{0} \\
& \mathbf{N}(\xi, u) \leq 0
\end{array}
$$

where $f(\xi, u)$ is the state equation of model (1), $\xi_{0}$ is the initial state vector, and $\mathbf{N}(\xi, u) \leq 0$ represents all state and input constraints in the vehicle system.

It is worth mentioning that the optimization problem (5), as formulated, includes no explicit guarantee of yaw stability. While a yaw rate constraint can be included in $\mathbf{N}(\xi, u)$, such a constraint was found to be largely inactive for an optimal solution, i.e. when $H_{\max }$ is minimized, the vehicle dynamics itself works to ensure adequate yaw stability. The same is true for a body sideslip constraint, which is also found to be redundant and therefore, for simplicity, not included.

\section{The MPC formulation}

The constrained nonlinear optimization problem (5) usually can be handled within the framework of Pontryagin's minimum principle, ${ }^{22}$ which will leads to the minimization of a Hamiltonian function and turns into a two-pointboundary-value-problem (TPBVP). Unless the system dynamics are quite simple, only off-line numerical solutions can be sought for the TPBVP problem with numerical approaches such as the shooting $\operatorname{method}^{23}$ or interior point method. ${ }^{24}$ Hence it is not generally applicable to implementing a real-time controller associated with a real driving task.

Though it is difficult to obtain an exact solution for the nonlinear optimization problem mentioned above, MPC can provide a feasible approach to obtaining near-optimal solutions usinga a discrete state space model. In what follows, we present the relevant formulation of MPC. This involves repeatedly solving a simplified optimization problem over a finite prediction horizon, taking account of the various actuator and friction constraints. ${ }^{25}$ A linear time varying (LTV) approximation of both the vehicle model (1) and the Pacejka tire model are used to predict the future evolution of the vehicle system. Essentially, the vehicle model is linearized at every sampling point using the current states and inputs. This leads to a LTV-MPC formulation, in which, a time-varying convex quadratic optimization problem is formulated and solved at each time step.

The vehicle model (1) can be written as:

$$
\dot{\xi}(t)=f(\xi, u)
$$

Additionally, we define the output equation to be:

$$
\eta(t)=c \cdot \xi(t)
$$

where

$$
c=\left[\begin{array}{llllll}
0 & 0 & 0 & 0 & 1 & 0 \\
0 & 0 & 0 & 0 & 0 & 1
\end{array}\right]
$$

i.e. output of the system is $\eta(t)=\left[\begin{array}{ll}X & Y\end{array}\right]^{T}$.

To obtain the LTV-MPC formulation for sampling instant $k$, denote the current measured states, inputs, and outputs as: $\xi^{k}, u^{k}$, and $\eta^{k}$ respectively (here and below superscripts or subscripts $k$ are used to denote the $k$ th sampling instant). Then the linearized system is:

$$
\begin{aligned}
\dot{\tilde{\xi}} & =A_{k} \cdot \tilde{\xi}+B_{k} \cdot \tilde{u} \\
\tilde{\eta} & =C_{k} \cdot \tilde{\xi}
\end{aligned}
$$


where

$$
\begin{aligned}
\tilde{\xi} & =\xi-\xi^{k}, \tilde{u}=u-u^{k}, \tilde{\eta}=\eta-\eta^{k}, \\
A_{k} & =\left.\frac{\partial f}{\partial \xi}\right|_{\left(\xi^{k}, u^{k}\right)}, \quad B_{k}=\left.\frac{\partial f}{\partial u}\right|_{\left(\xi^{k}, u^{k}\right)} \\
C_{k} & =\left.\frac{\partial \eta}{\partial \xi}\right|_{\left(\xi^{k}, u^{k}\right)}=\left[\begin{array}{cccccc}
0 & 0 & 0 & 0 & 1 & 0 \\
0 & 0 & 0 & 0 & 0 & 1
\end{array}\right]
\end{aligned}
$$

The linearized model (6) is further discretized as following:

$$
\begin{aligned}
\tilde{\xi}(k+1) & =A_{k}^{d} \cdot \tilde{\xi}(k)+B_{k}^{d} \cdot \tilde{u}(k) \\
\tilde{\eta}(k) & =C_{k}^{d} \cdot \tilde{\xi}(k)
\end{aligned}
$$

where $A_{k}^{d}, B_{k}^{d}, C_{k}^{d}$ are system matrices of the discrete system.

The LTV-MPC uses the discrete model (7) as its prediction model and this model updates at every sampling instant. The remaining formulation of MPC adopts a similar procedure as in the standard MPC literature. ${ }^{13 ; 14 ; 25}$ The crucial point here is to choose a suitable cost function for MPC to minimize, one that can reflect the objective of the offtracking minimization problem (5), as well as defining a set of problem-specific constraints.

Let the prediction horizon be $H_{p}$, and the control horizon be $H_{c}$, where typically we have $H_{p} \geq H_{c}$. The optimization problem of the LTV-MPC is defined to be:

$$
\begin{aligned}
\min _{\Delta \tilde{u}(k+i \mid k)} J= & \sum_{i=1}^{H_{p}}\left\|\tilde{\eta}(k+i \mid k)-\tilde{\eta}_{r e f}(k+i \mid k)\right\|_{Q}^{2} \\
& +\sum_{i=0}^{H_{c}-1}\|\Delta \tilde{u}(k+i \mid k)\|_{R}^{2}
\end{aligned}
$$

subject to

$$
\begin{aligned}
& \tilde{\xi}(k+1)=A_{k}^{d} \cdot \tilde{\xi}(k)+B_{k}^{d} \cdot \tilde{u}(k) \\
& \tilde{\eta}(k)=C_{k}^{d} \cdot \tilde{\xi}(k) \\
& \tilde{u}(k+i)=\tilde{u}(k+i-1)+\Delta \tilde{u}(k+i)
\end{aligned}
$$

$$
\begin{aligned}
& \Delta u_{\min } \leq \Delta \tilde{u} \leq \Delta u_{\max } \\
& u_{\min } \leq \tilde{u} \leq u_{\max } \\
& \xi_{\min } \leq \xi \leq \xi_{\max }
\end{aligned}
$$

where $Q=\operatorname{diag}\left[q_{x}, q_{y}\right]$ is a positive definite matrix that weights the distance travelled in the $X, Y$ directions. $R=\operatorname{diag}\left[r_{f l}, r_{f r}, r_{r l}, r_{r r}\right]$ is a positive definite matrix weighting the inputs, i.e. four brake forces of the wheels. Equation (8) defines the objective function to be minimized. It includes two terms, the first being the weighted sum of difference from vehicle's current position to the reference position, and the second is the weighted sum of incremental inputs. To make this objective function reflect the optimization problem (5), we choose the reference position to be the center of the curved path. Since the model (7) is linearized around $\xi^{k}, u^{k}$, and $\eta^{k}$, the position error should be:

$$
\tilde{\eta}_{r e f}(k+i \mid k)=\left[\begin{array}{ll}
0 & Y_{M}
\end{array}\right]^{T}-\eta^{k}, \quad \forall i=1,2, \cdots, H_{p}
$$

Equations (9) and (10) represent the system dynamics while equation (11) defines the incremental inputs to be used in the MPC scheme. Then equations (12), (13) and (14) capture the input and state constraints. Note that in this particular application, we did not apply constraints on the variation of control inputs and the states, i.e. $\Delta u_{\min }, \Delta u_{\max }, \xi_{\min }, \xi_{\max }$ are not applicable here. On the other hand, constraints on the magnitude of control inputs must be considered to reflect the permissable range of the braking forces. Thus $u_{\max }$ and $u_{\min }$ are given as follows:

$$
u_{\max }=\left[\begin{array}{c}
0 \\
0 \\
0 \\
0
\end{array}\right], u_{\min }=\left[\begin{array}{c}
-F_{z f l} \cos \alpha_{f l} \\
-F_{z f r} \cos \alpha_{f r} \\
-F_{z r l} \cos \alpha_{r l} \\
-F_{z r r} \cos \alpha_{r r}
\end{array}\right] .
$$

These constraints ensure that only braking forces are applied, and with maximum force corresponding to the locked wheel condition. The MPC scheme then translates the problem defined by equation (8) to Equation (14) into a quadratic programming $(\mathrm{QP})$ problem, from which an optimal control sequence $\Delta \tilde{u}(k+i \mid k), i=0,2, \cdots, H_{c}-1$ can be solved. And according to the receding horizon principle, only the first control is applied at the current step. In the next sampling instant, the whole procedure is repeated.

\section{Simulation results}

To evaluate the performance, the MPC braking controller described in the previous section has been implemented in co-simulation with two different commercial nonlinear vehicle simulation packages, CarSim 8.1 and CarMaker 5.0. CarSim was used to validate the control software, but since the tyre model was carefully fitted to that vehicle model, it was considered important for robustness to use an alternative vehicle simulation environment, i.e. CarMaker, used as a surrogate for a real test vehicle.

The test scenario is a circular path with radius of 60 meters, and the friction coefficient was set to 0.4 , which represents a relatively low-mu surface such as packed snow 


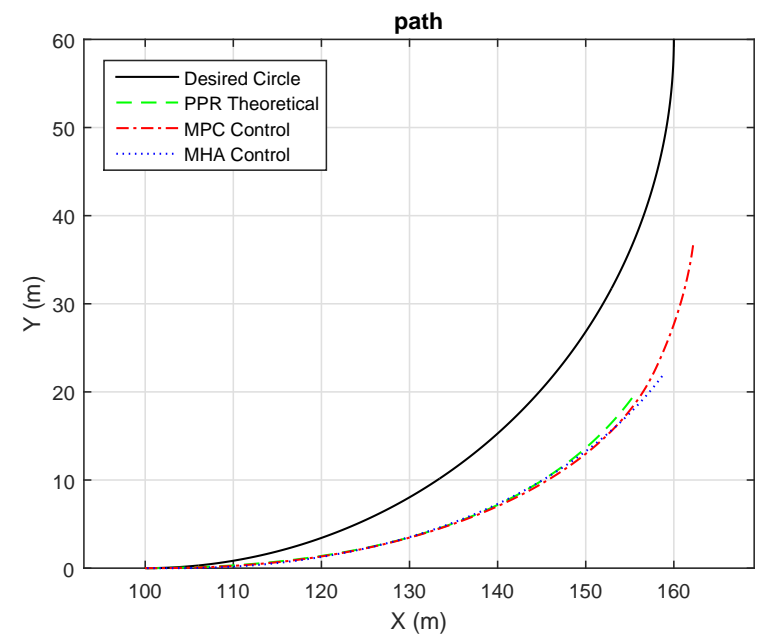

Figure 6. Comparisons of the vehicle path with the desired circle, the PPR theoretical path and MHA control

or wet concrete. From the settings, we determine:

$$
v_{\text {lim }}=\sqrt{\mu g R_{\text {ref }}}=\sqrt{0.4 \times 9.81 \times 60}=15.344(\mathrm{~m} / \mathrm{s})
$$

The vehicle's initial speed was set to $v_{0}=20(\mathrm{~m} / \mathrm{s})$, so for $v_{0}>v_{\text {lim }}$, the MPC brake controller is activated to mitigate road departure. The vehicle type used in the simulation is an E-class big sedan. The main vehicle parameters as well as the controller parameters are listed in Table 2.

Table 2. Main vehicle parameters and controller parameters

\begin{tabular}{lll}
\hline Description & Symbol & Value \\
\hline Vehicle mass & $m$ & $1572(\mathrm{~kg})$ \\
Yaw moment of inertia & $I_{z z}$ & $2634\left(\mathrm{~kg} \cdot \mathrm{m}^{2}\right)$ \\
Wheelbase & $L$ & $2.79(\mathrm{~m})$ \\
Distance to CG (front axle) & $l_{f}$ & $1.357(\mathrm{~m})$ \\
Distance to CG (rear axle) & $l_{f}$ & $1.433(\mathrm{~m})$ \\
Steering ratio & $i_{S}$ & 16 \\
Step-steer magnitude & $\delta_{H}$ & $42.628(\mathrm{deg})$ \\
Half track & $l_{t}$ & $0.782(\mathrm{~m})$ \\
Tire effective rolling radius & $R_{t}$ & $0.31(\mathrm{~m})$ \\
Road friction coefficient & $\mu$ & 0.4 \\
Radius of desired path & $R_{r e f}$ & $60(\mathrm{~m})$ \\
Gravity constant & $g$ & $9.81\left(\mathrm{~m} / \mathrm{s}^{2}\right)$ \\
Prediction horizon & $H_{p}$ & 10 \\
Control horizon & $H_{c}$ & 10 \\
Sampling period & $T_{s}$ & $0.1(\mathrm{~s})$ \\
Output weights on $X$ & $q_{x}$ & 34.8518 \\
Output weights on $Y$ & $q_{y}$ & 20.8464 \\
Input weights & $r_{* *}$ & 0.001 \\
\hline
\end{tabular}

The simulation results are illustrated in Figure 6 to Figure 11.

Figure 6 illustrates the vehicle's actual path as well as comparisons with the desired circular path, the PPR theoretical path and also the path of MHA control, a controller used previously in the literature to solve the same control problem. ${ }^{10-12}$ It shows that the PPR, MPC and MHA paths all deviate from the desired circle, due to the $v_{0}>$ $v_{\text {lim }}$ condition. It is known that PPR provides a global
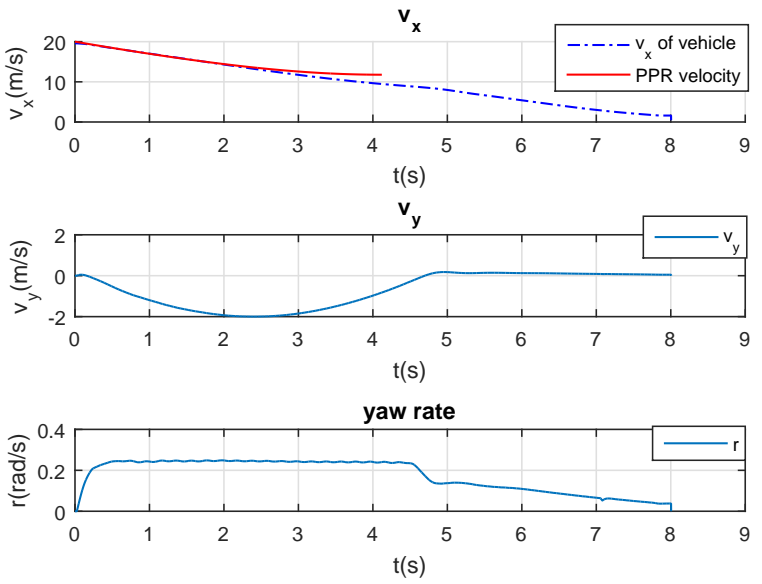

Figure 7. Longitudinal, lateral speed and yaw rate
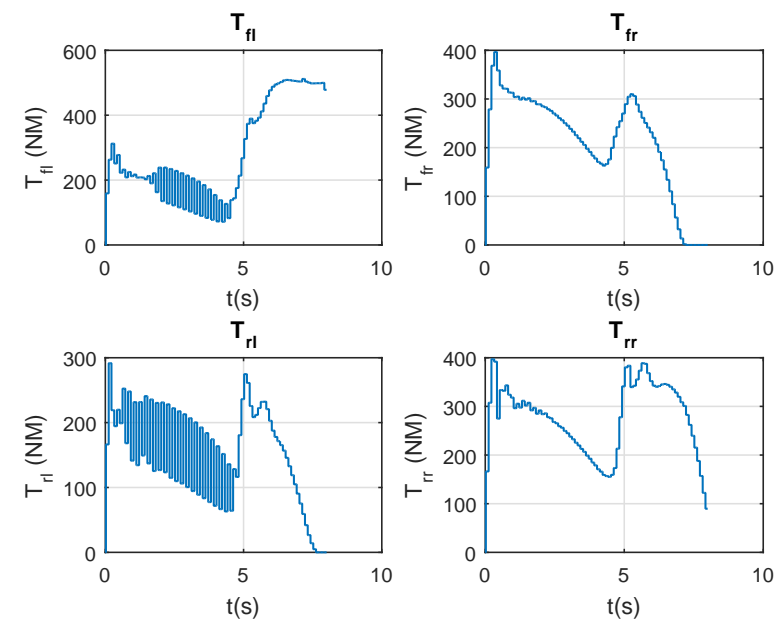

Figure 8. Brake torques applied on four wheels
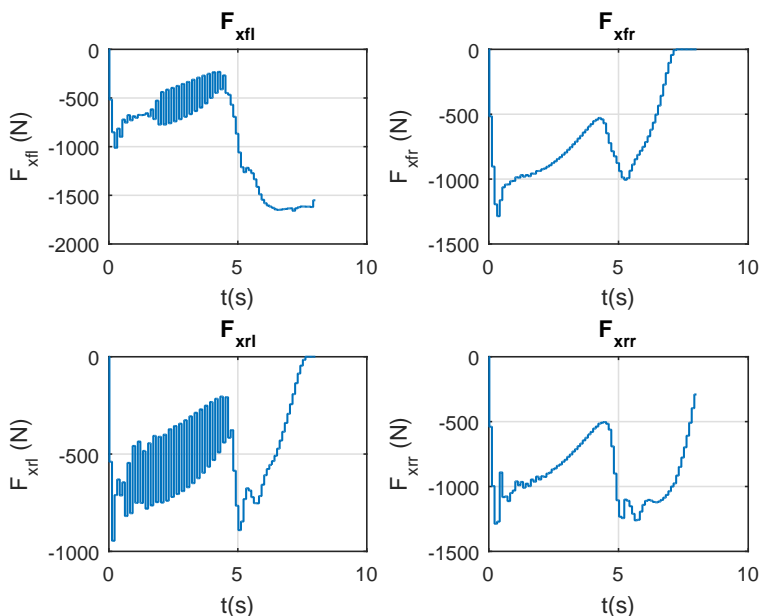

Figure 9. Longitudinal tire forces of four wheels

optimum solved from the Pontryagin's minimum principle by assuming the vehicle is a free particle model, hence it is not realistically possible to achieve better results than PPR, except by increasing the surface friction or performing some 

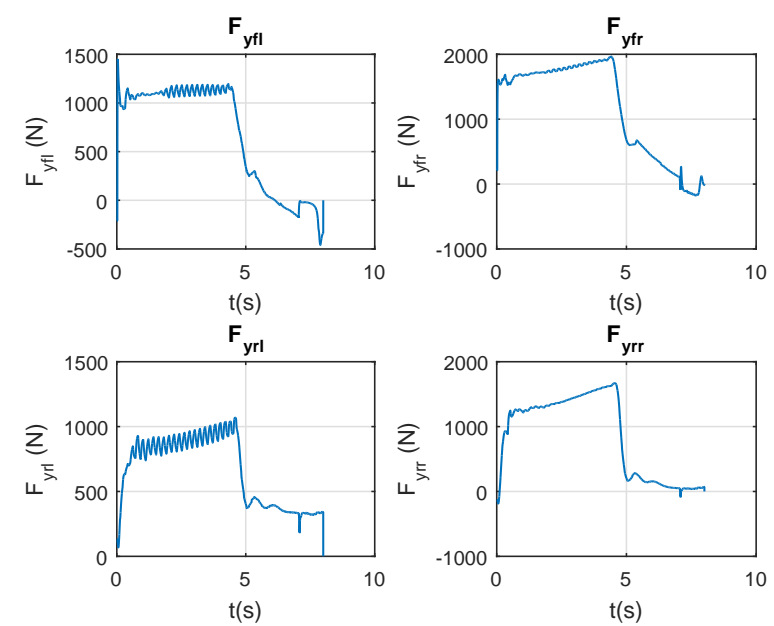

Figure 10. Lateral tire forces of four wheels
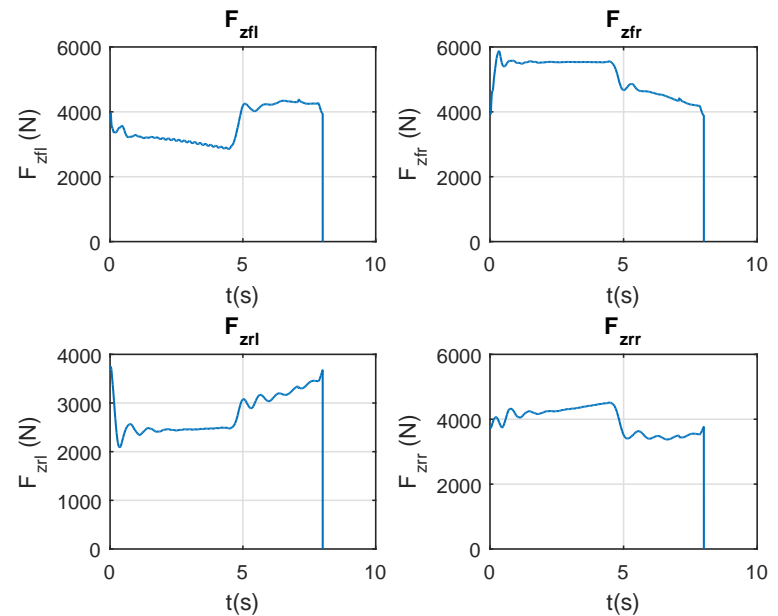

Figure 11. Vertical loads of four wheels

change in initial path or speed. The figure shows that both the MPC and MHA path are very close to the PPR path, and the MPC and MHA paths are very close to each other as well, confirming that both has solved the common optimization problem, albeit using entirely different techniques. Overall we conclude that MPC control is able to obtain solution that is very close to optimal, and is comparable to MHA. Figure 7 illustrates the longitudinal velocity, lateral velocity and yaw rate; the longitudinal velocity with MPC control decreases in very similar fashion to the PPR solution. The magnitudes of the lateral velocity and yaw rate are relatively small and both converge to zero as time elapses, which indicates the vehicle has good stability for the optimized path.

Figure 8 shows the brake torque generated by the MPC controller at each of the four wheels. The front left and rear left wheel brake torque shows extensive chattering for earlier periods in order to achieve optimization. This may be be suppressed by increasing the penalty factor $r_{* *}$ in the cost function $J$ or imposing an additional constraint on $\Delta \tilde{u}$, but both will deteriorate the optimal performance. So, to explore the maximal potential that MPC can achieve, we prefer not to suppress this fast switching phenomenon. In general, we can see that in the early stage, when the longitudinal speed is high, the outer wheels brake harder than the inner wheels. This indicates that the MPC controller prioritizes hard braking on the more heavily loaded (outer) wheels leading to a turning-out yaw moment, in contradiction to the classic turning-in yaw moment of conventional ESC systems. This same effect was seen in other research. ${ }^{9}$ Meanwhile this effect can enhance lateral stability by avoiding large body side slip. In the later braking phase, as the speed decreases, the inner wheels progressively brake more compared with the outer wheels, showing that the MPC controller progressively shifts its bias towards cornering acceleration.

Figures 9 to Figure 11 illustrate the longitudinal, lateral and vertical tyre forces. Figure 9 also shows chattering since longitudinal tire forces are directly related to the brake torques. Figure 10 shows much smaller chattering in the lateral forces of four wheels, most likely due to the tire structure absorbing or filtering the vibrational excitation. Figure 11 shows the dynamic load transfer effects of the vehicle, where as expected the outer wheels have increased vertical load compared with the inner wheels. More specifically, during combined cornering to left and simultaneously braking, the front right wheel experiences the greatest load and the rear left wheel has the smallest load, which is in line with intuition and common experience.

The online computational burden of the proposed MPC control scheme mainly lies in finding the solution of the resulting QP problem. In the above work, the Matlab function quadprog was used for solving the QP problem. Additional computational burden comes from linearization, discretization and translation of the problem (8) into a QP problem. The exact computational cost largely depends on the dimensions of the system states and outputs, as well as the magnitudes of the prediction and control horizons. Here the system dimensions are fixed while the prediction and control horizons can be adjusted. With the controller settings in Table 2, it is found that the CarMaker simulation runs faster than real-time on a PC with an Intel i5 CPU, running Windows 7 and Matlab R2015a. This broadly suggests that the current implementation of MPC has the potential to be used as a real-time control method.

\section{Conclusion}

This paper has considered the problem of reducing the lateral path deviation of higher speed vehicles on a low 
friction surface via an MPC control scheme. The paper provides new and independent confirmation of the validity of the PPR control model, combining speed reduction and path curvature control as a means of terminal understeer mitigation. More significantly, it demonstrates that MPC is a viable tool for road vehicle control at the limits of friction. The optimal solution from MPC confirms earlier studies where there is a somewhat counter-intuitive action of braking the outer wheels harder than the inner wheels at high speed, in contrast to the more conventional ESC formulation of providing a turn-in yaw moment. The MPC approach was applied to a 'known' problem, but it is expected that the same approach can be used in more complex scenarios, for example in automated collision avoidance involving traffic or other moving objects. Overall, the novelty and contribution of this paper can be summarized in the following aspects:

1. In the absence of cost terms designed to suppress chattering, an ABS-like switching phenomenon emerges from real-time optimization designed to achieve a minimum lane or departure. While it cannot be concluded that this is a general result, it seems to arise quite naturally from the control problem. We note that the same behavior was also discovered by other optimal control methods including MHA.

2. The formulation of MPC in the current paper uses an online linearization concept that fully adapts to the changing operation point close to the friction limit. Moreover, while similar published research makes use of the simple bicycle model, the work has employed a two-track vehicle model; the effects of load transfer clearly seen in the results - is an important reason to make use of a two track model for MPC operating in the nonlinear handling region.

3. The proposed control approach is validated via commercial simulation packages with high fidelity vehicle models.

4. The results confirm earlier research results derived by different methods, providing important crossvalidation. And the generality of MPC suggests the approach will be applicable to large number of new scenarios with complex tire models operating right up to the limits of friction.

5. The control method appears robust to assumptions about the detailed tyre characteristics, insofar as the controller design did not make use of the precise parameters (especially tyre parameters) of the model used for validation. The method introduced a tyre model with an elliptical relationship between $F_{x}$ and
$F_{y}$ rather than the simple friction circle or linear tire models used in earlier research.

\section{References}

1. Galvani M, Biral F and Fujimoto H. Four wheel optimal autonomous steering for improving safety in emergency collision avoidance manoeuvres. In: 13th IEEE international workshop on advanced motion control (AMC), Yokohama, Japan, 14-16 March 2014, pp. 362-267.

2. Liu C and Subramanian R. Factors related to fatal singlevehicle run-off-road crashes. Report NHTSA DOT HS 811 232, National Highway Traffic Safety Administration, US Department of Transportation, Washington, DC, USA, 2009.

3. Liu $C$ and Ye TJ. Run-off-road crashes: an on-scene perspective. Report NHTSA DOT HS 811 500, National Highway Traffic Safety Administration, US Department of Transportation, Washington, DC, USA, 2011.

4. Shibahata Y, Shimada K and Tomari T. Improvement of vehicle maneuverability by direct yaw moment control. Vehicle System Dynamics 1993; 22: 465-481.

5. Ghoneim YA, Lin WC, Sidlosky DM, et al. Integrated chassis control system to enhance vehicle stability. Int J Veh Des 2000; 23: 124-144.

6. Tseng HE, Ashrafi B, Madau D, et al. The development of vehicle stability control at ford: Focused section on mechatronics in automotive systems. IEEE/ASME Trans. on Mechatronics 1999; 4: 223-234.

7. Takahashi J, Yamakado M, Saito S, et al. A hybrid stabilitycontrol system: combining direct-yaw-moment control and gvectoring control. Vehicle System Dynamics 2012; 50: 847-859.

8. Gordon T, Klomp M and Lidberg M. Control mitigation for over-speeding in curves: Strategies to minimize off-tracking. In: 11th international symposium on advanced vehicle control (AVEC'12), Seoul, Korea, 9-12 September 2012.

9. Klomp M, Lidberg $\mathrm{M}$ and Gordon T. On optimal recovery from terminal understeer. Proc. Inst. Mech. Eng., Part D, J. Automobile Eng. 2014; 228: 412-425.

10. Gao Y, Gordon T, Lidberg M, et al. An autonomous safety system for road departure prevention based on combined path and sideslip control. In: 24th symposium of the international association for vehicle system dynamics (IAVSD), Graz, Austria, 17-21 August 2015, pp. 281-286.

11. Gao Y, Gordon $\mathrm{T}$ and Lidberg M. Implementation of a modified hamiltonian algorithm for control allocation. In: Proceedings of 13th international symposium on advanced vehicle control (AVEC'16), Munich, Germany, 13-16 Sept 2016.

12. Zhang D, Gordon $\mathrm{T}, \mathrm{Gao} \mathrm{Y}$ and Zong $\mathrm{C}$. Intelligent electronic steering program based on road departure mitigation control. 
In: Proceedings of the 2016 IEEE international conference on systems, man, and cybernetics (SMC 2016), Budapest, Hungary, 9-12 Oct 2016.

13. Falcone P, Borrelli F, Asgari J, et al. Predictive active steering control for autonomous vehicle systems. IEEE Transactions on Control Systems Technology 2007; 15: 566-580.

14. Chang $\mathrm{S}$ and Gordon T. A flexible hierarchical model-based control methodology for vehicle active safety systems. Vehicle System Dynamics 2008; 46: 63-75.

15. Di Cairano S, Tseng E, Bernardini D, et al. Steering vehicle control by switched model predictive control. In: 6 th IFAC symposium "advances in automotive control", Munich, Germany, 12-14 July 2010, pp. 1-6.

16. Shim T, Adireddy $G$ and Yuan H. Autonomous vehicle collision avoidance system using path planning and modelpredictive-control-based active front steering and wheel torque control. Proc. Inst. Mech. Eng., Part D, J. Automobile Eng. 2012; 226: 767-778.

17. Attia R, Orjuela R and Basset M. Combined longitudinal and lateral control for automated vehicle guidance. Vehicle System Dynamics 2014; 52: 261-279.

18. Liu X, Li M and Xu M. Model-predictive-control-based novel anti-skid method for electric vehicles using the wheel acceleration and the motor torque. Proc. Inst. Mech. Eng., Part D, J. Automobile Eng. 2016; 230: 1780-1790.

19. Choi M and Choi S. MPC for vehicle lateral stability via differential braking and active front steering considering practical aspects. Proc. Inst. Mech. Eng., Part D, J. Automobile Eng. 2016; 230: 459-469.

20. Pacejka HB. Tyre and vehicle dynamics. 2nd edition. Oxford: Butterworth-Heinemann, 2006.

21. Ann Arbor. CarSim Reference Manual, Ver 8.0. Michigan: Mechanical Simulation Corporation, 2009.

22. Naidu DS. Optimal Control Systems. CRC Press, 2002.

23. Stoer J and Bulirsch R. Introduction to Numerical Analysis. New York: Springer-Verlag, 2002.

24. Potra F and Wright S. Interior-point methods. Journal of Computational and Applied Mathematics 2000; 124(1-2):281302.

25. Wang L. Model predictive control system design and implementation using MATLAB. Springer-Verlag London, 2009. 